\title{
Microvesicles shed from bortezomib-treated or lenalidomide-treated human myeloma cells inhibit angiogenesis in vitro
}

\author{
HUI-MEI GUO, LI SUN, LIN YANG, XIAO-JUN LIU, ZI-YUAN NIE and JIAN-MIN LUO \\ Department of Hematology, The Second Hospital of Hebei Medical University, \\ Key Laboratory of Hematology, Shijiazhuang, Hebei 050000, P.R. China
}

Received November 18, 2017; Accepted April 17, 2018

DOI: $10.3892 /$ or.2018.6395

\begin{abstract}
Angiogenesis plays a significant role in the pathogenesis of multiple myeloma (MM). Microvesicles (MVs), a type of extracellular vesicles, are known as important players in cell-to-cell communication. MM-derived MVs have exhibited the activity of promoting angiogenesis. Bortezomib and lenalidomide are important drugs for treating myeloma. Therefore, the aim of the present study was to investigate whether and how MVs secreted from human myeloma cells exposed to bortezomib and lenalidomide affect angiogenesis. RPMI-8226 human myeloma cells and human umbilical vein endothelial cells (HUVECs) were used. MVs were isolated from the drug-treated RPMI-8226 cells. The number of the MVs were analyzed with flow cytometry. The expression of pro-angiogenic factors was analyzed with PCR and ELISA. The angiogenic potential of HUVECs was examined. NF- $\kappa \mathrm{B}$ activation was analyzed using PCR, immunofluorescent staining and western blotting assays. We showed that bortezomib treatment induced an increase in the number of MVs shed from myeloma cells, but the number of MVs was not significantly altered by lenalidomide. The expression levels of vascular endothelial growth factor (VEGF), interleukin 6 (IL-6) and basic fibroblast growth factor (bFGF) were reduced in the MVs from the RPMI-8226 cells exposed to bortezomib and lenalidomide. Consequently, these MVs exhibited reduced angiogenic potential, as evaluated by wound healing tests, Boyden chamber assays and tube formation assays. Co-culturing HUVECs with drug-treated MVs inhibited $\mathrm{NF}-\kappa \mathrm{B}$ activation in the HUVECs and reduced the secretion of pro-angiogenic factors. In conclusion, bortezomib and
\end{abstract}

Correspondence to: Dr Jian-Min Luo, Department of Hematology, The Second Hospital of Hebei Medical University, Key Laboratory of Hematology, 215 West Heping Road, Shijiazhuang, Hebei 050000, P.R. China

E-mail: luojm2011@163.com

Key words: multiple myeloma, microvesicles, bortezomib, lenalidomide, angiogenesis, $\mathrm{NF}-\kappa \mathrm{B}$ lenalidomide treatment of cultured myeloma cells can block $\mathrm{MV}$-induced angiogenesis and hence provides another mechanism for anti-angiogenic therapy.

\section{Introduction}

Multiple myeloma (MM) is an incurable hematologic neoplasm of plasma cells. Despite the emergence of novel drugs including proteasome inhibitors and immunomodulating drugs (IMiDs) that have improved the overall patient outcome (1), the prognosis for MM patients is still poor, and the pathogenesis of this disease is only partially understood. Angiogenesis plays a significant role in the pathogenesis of MM (2). Recent data have shown that there is a close relationship between MM cells and adjacent cells in the microenvironment (3) and that angiogenesis in $\mathrm{MM}$ is tightly controlled by angiogenic cytokines and inflammatory factors $(4,5)$. However, how the release of these cytokines is regulated and how myeloma cells act on microvascular endothelial cells and bone marrow stromal cells to promote angiogenesis remain unclear.

Microvesicles (MVs), a type of extracellular vesicles (EVs), are also called shedding vesicles or microparticles (MPs) and have emerged as important players in cell-to-cell communication (6-8). MVs can load and transmit bioactive molecules to target cells (9-11) and induce different signaling cascades $(10,12,13)$. A previous study found that MVs derived from MM cells (MM MVs) can be internalized by endothelial cells and promote angiogenesis (14). However, the mechanism by which MM MVs promote endothelial cell angiogenesis remains unclear.

The NF- $\mathrm{BB}$ signaling pathway is constitutively active in $\mathrm{MM}$ and regulates the expression of numerous genes, such as vascular endothelial growth factor (VEGF) and interleukin 6 (IL-6), that are involved in the pathogenesis of MM including angiogenesis (15-17). Bortezomib and lenalidomide are important drugs for treating myeloma, and both of them block NF- $\mathrm{B}$ activation (18-20). Some studies have examined the MVs shed by cells after drug treatments. One study demonstrated that tumor-derived microparticles (TMPs) from mammary carcinoma cells exposed to paclitaxel chemotherapy contributed to angiogenesis and tumor regrowth (21). However, another study showed that the neutralization of VEGF-A 
with anti-angiogenic drugs in cultured breast carcinoma cells blocked TMP-induced angiogenesis (22). There has been little research on the effect of drugs on MM MVs. A recent study (23) showed that the pro-angiogenic activity of MVs derived from bortezomib-treated myeloma cells was decreased, but the molecular mechanisms behind this inhibition of angiogenesis have not yet been elucidated. Moreover, there have been no reports on the effect of IMiDs, including thalidomide and lenalidomide, on the pro-angiogenic activity of MM MVs.

In the present study, we determined whether and how bortezomib and lenalidomide affect the characteristics of MM MVs derived from RPMI-8226 cells, including their number and expression of pro-angiogenic factors and their effects on the migration, invasion and tube formation of endothelial cells. In this context, we evaluated the expression levels of pro-angiogenic factors and the activity of $\mathrm{NF}-\kappa \mathrm{B}$ in endothelial cells.

\section{Materials and methods}

Cell lines and culture. The RPMI-8226 human multiple myeloma cell line and human umbilical vein endothelial cells (HUVECs) were purchased from the American Type Culture Collection (ATCC, Manassas, VA, USA). The cells were cultured in medium containing $10 \%$ fetal bovine serum (FBS, Gibco; Thermo Fisher Scientific, Inc., Waltham, MA, USA) and $1 \%$ penicillin-streptomycin (Solarbio, Beijing, China) at $37^{\circ} \mathrm{C}$ with $5 \% \mathrm{CO}_{2}$.

$M V$ isolation. MVs were isolated by continuous differential centrifugation as previously described (24) from the different conditioned media of myeloma cells. Briefly, MM cells growing in $\log$ phase were seeded at $5 \times 10^{5}$ cells $/ \mathrm{ml}$ and were randomly divided into 5 groups depending on treatment: control group (PBS), 10 or $100 \mathrm{nM}$ bortezomib (Selleck Chemicals, Houston, TX, USA), 1 or $10 \mu \mathrm{M}$ lenalidomide (MedChem Express, Monmouth Junction, NJ, USA). MVs derived from corresponding RPMI-8226 cells from the 5 groups above were named: N-MV (PBS), B10-MV (10 nM bortezomib), B100-MV (100 nM bortezomib), L1-MV (1 $\mu \mathrm{M}$ lenalidomide), and L10-MV (10 $\mu \mathrm{M}$ lenalidomide). After $24 \mathrm{~h}$ of culture, the conditioned media were centrifuged at $750 \times \mathrm{g}$ for $5 \mathrm{~min}$ and then at $1500 \mathrm{x}$ g for $20 \mathrm{~min}$ to remove cells and debris. After further centrifugation at $16000 \mathrm{x} \mathrm{g}$ for $45 \mathrm{~min}$ at $4^{\circ} \mathrm{C}$, the MVs were pelleted. After washing twice with PBS, the pelleted MVs were resuspended in PBS, stored at $4^{\circ} \mathrm{C}$ and used for experiments within a few days.

Flow cytometric analysis of MVs. The number of MVs was determined by flow cytometry (CytoFLEX, Beckman Coulter, Inc., Brea, CA, USA) following the manufacturer's protocols. Standard microbeads (1.1 $\mu \mathrm{m}$, Sigma-Aldrich; Merck KGaA, Darmstadt, Germany) were used for size calibration, and the number of MVs were calculated using 3- $\mu \mathrm{m}$ standard microbeads (Sigma-Aldrich; Merck KGaA) as previously described (24). Briefly, 1.1- $\mu \mathrm{m}$ standard microbeads were used to set the gate, $3-\mu \mathrm{m}$ standard microbeads of an equal concentration were added to the sample as an internal standard, and the counting endpoint was set at 100,000 events for the $3-\mu \mathrm{m}$ standard bead population.
Quantitative real-time polymerase chain reaction $(q P C R)$ and enzyme-linked immunosorbent assay (ELISA). To evaluate the expression levels of pro-angiogenic factors, using qPCR, we analyzed the mRNA expression levels of VEGF, IL-6 and basic fibroblast growth factor (bFGF) in different groups of MM MVs and HUVECs, and by ELISA, we analyzed the protein expression levels of these factors in the conditioned medium of HUVECs. Additionally, we measured the mRNA expression of $\mathrm{NF}-\kappa \mathrm{B}$ in HUVECs. MM MVs from the 5 different groups were isolated and subjected to RNA preparation using TRIzol (Invitrogen; Thermo Fisher Scientific, Inc.) and to cDNA synthesis with a cDNA Synthesis kit (Thermo Fisher Scientific, Inc.) according to the manufacturer's protocol. The total RNA of HUVECs $\left(5 \times 10^{4}\right.$ cells $\left./ \mathrm{ml}\right)$ co-cultured for $24 \mathrm{~h}$ with MM MVs $(10 \mu \mathrm{g} / \mathrm{ml})$ from the 5 different groups above or without MVs was isolated, and cDNA was synthesized. Each qPCR reaction was prepared in triplicate. PCR was performed using SuperReal PreMix Plus (SYBR Green) (Tiangen, Beijing, China) with a real-time PCR system (Rotor-Gene Q, Qiagen, Hilden, Germany). The following primer sequences (Invitrogen; Thermo Fisher Scientific, Inc.) were used: VEGF, 5'-AATGCTTTCT CCGCTCTGAA-3'(F) and 5'-GCTTCCTACAGCACAGC AGA-3' (R) (96 bp); IL-6, 5'-ATGAGGAGACTTGCCTGGTG AAAAT-3' (F) and 5'-TCTGGCTTGTTCCTCACTACT-3' (R) (104 bp); bFGF, 5'-TTCATAGCAAGGTACCGGTTG-3' (F) and 5'-AGAAGAGCGACCCACACG-3' (R) (97 bp); NF-кB, 5'-GGGGACTACGACCTGAATG-3' (F) and 5'-GGGCACG ATTGTCAAAGAT-3' (R) (118 bp); and $\beta$-actin, 5'-GAGCT ACGAGCTGCCTGAC-3' (F) and 5'-GGTAGTTTCGTG GATGCCACAG-3' (R) (121 bp). The qPCR conditions were $2 \mathrm{~min}$ at $50^{\circ} \mathrm{C}, 2 \mathrm{~min}$ at $95^{\circ} \mathrm{C}$ and then 40 cycles of $15 \mathrm{sec}$ at $95^{\circ} \mathrm{C}$ and $30 \mathrm{sec}$ at $60^{\circ} \mathrm{C}$. A comparative $\Delta \mathrm{Ct}$ method was used to determine gene expression and $\beta$-actin served as the normalization gene.

The expression levels of human VEGF, IL- 6 and bFGF in the supernatants of HUVECs were measured by ELISA kits (NeoBioscience, Shenzhen, China) according to the manufacturer's instructions. Experiments were performed in triplicate.

\section{Angiogenesis assays}

Wound healing assay. Cell migration was determined using a scratch adhesion test. Briefly, the outside undersurface of a 6-well plate was scored with a marker pen; HUVECs $\left(5 \times 10^{5}\right.$ cells/well) were seeded onto a 6 -well plate and cultured overnight to confluence. Then, at time zero, the cell monolayer was wounded artificially across the marked line using a 200- $\mu 1$ pipette tip. The scratched area was captured using an inverted microscope (Axio Observer D1, Zeiss, Germany) in lowpower fields (x40). Then, the cells were treated with medium (DMEM without FBS) and the 5 groups of MVs $(10 \mu \mathrm{g} / \mathrm{ml})$ for $24 \mathrm{~h}$. Negative control cells were treated with PBS. The wounded area was captured again at $24 \mathrm{~h}$ and measured by ImageJ software (National Institutes of Health, Bethesda, MD, USA). The percentage of recovery was assessed. The experiment was repeated in triplicate, and each sample was tested in quadruplicate.

Cell invasion assay. Endothelial cell invasiveness was investigated using Transwell Boyden chambers $(6.5 \mathrm{~mm}$, Costar, 
USA). The polycarbonate membrane ( $8-\mu \mathrm{m}$ pore size) was coated with a layer of Matrigel matrix (Corning Inc., Corning, NY, USA). HUVECs ( $2 \times 10^{5}$ cells per well) were added to the upper chamber and starved; $750 \mu \mathrm{l}$ of complete medium with the indicated group of MVs was added at the indicated concentration $(50 \mu \mathrm{g} / \mathrm{ml})$ to the lower compartment of the chamber as a chemoattractant. PBS was used as the negative control. After incubation at $37^{\circ} \mathrm{C}$ for $6 \mathrm{~h}$, the cells on the bottom surface of the membrane were fixed with methanol, stained with Diff-Quik (Nanjing Jiancheng Bioengineering Institute, Nanjing, China) and counted in five representative high-power fields (x400).

Tube formation assay. Matrigel (50 $\mu 1$, Corning Inc.) was added to each well of a 96-well plate and allowed to polymerize at $37^{\circ} \mathrm{C}$ for $30 \mathrm{~min}$. Then, HUVECs $\left(2 \times 10^{4}\right.$ cells per well $)$ were cultured in DMEM with 6\% FBS in the presence of the 5 groups of MVs $(10 \mu \mathrm{g} / \mathrm{ml})$. PBS was used as the negative control. Each treatment was repeated in three wells. After incubation for 6 and $24 \mathrm{~h}$, images of the tubular structure that formed in each well were captured with an inverted microscope (Axio Observer D1, Zeiss, Germany) with an attached digital camera (Axiocam 506 color, Zeiss, Germany). The closed tube formation was quantified in five low-power fields ( $\mathrm{x} 40)$.

Immunofluorescent (IF) measurement of $N F-\kappa B$. HUVECs were seeded on climbing slices in 24-well plates and co-cultured with the 5 groups of MM MVs or PBS for $24 \mathrm{~h}$. To measure the effect of $\mathrm{NF}-\kappa \mathrm{B}$ activation, an NF- $\kappa \mathrm{B}$ Activation Nuclear Translocation assay kit (Beyotime Biotech, Haimen, China) was used according to the manufacturer's protocol and a previous study (25). Nuclei (blue) and NF- $\kappa \mathrm{B}$ (red) were viewed with a fluorescence-equipped microscope (BX51, Olympus, Tokyo, Japan). Co-localization of NF- $\kappa$ B with the nucleus appeared purple.

Western blot analysis. After co-culture with one of the 5 different MVs at $10 \mu \mathrm{g} / \mathrm{ml}$ or with PBS for $24 \mathrm{~h}$, nuclear protein or total protein were extracted from HUVECs using a Nuclear Protein Extraction kit (BestBio, Shanghai, China) or a Total Protein Extraction kit (BestBio), respectively, and then quantified by the BCA method. Equal amounts $(50 \mu \mathrm{g})$ of protein extracts were loaded and separated by SDS-PAGE and then transferred electrophoretically to a polyvinylidene difluoride (PVDF) membrane. Nonspecific sites were blocked by incubation with $5 \%$ nonfat dry milk in TBST at room temperature for $1 \mathrm{~h}$. The membranes were incubated with the indicated primary antibodies [P65 (1:50000, 7R200963-9, Abcam, Cambridge, MA, USA, rabbit monoclonal), p-p65 (1:1000, 3033S, Cell Signaling Technology, Inc., Danvers, MA, USA; rabbit monoclonal), H3 (1:2000, KM9005T, Tianjin Sungene Biotech, Tianjin, China; mouse monoclonal), and $\beta$-actin (1:1000, sc-130657, Santa Cruz Biotechnology, Santa Cruz, CA, USA; rabbit polyclonal)] in blocking buffer overnight at $4^{\circ} \mathrm{C}$. Anti-rabbit or mouse $\operatorname{IgG}(1: 10000,21032$, Rockland, Limerick, PA, USA) was used as a secondary antibody and incubated with the membrane for $2 \mathrm{~h}$ at room temperature. After washing, reactive bands were identified by scanning infrared laser microscopy (LI-COR Biosciences, Lincoln, NE, USA).

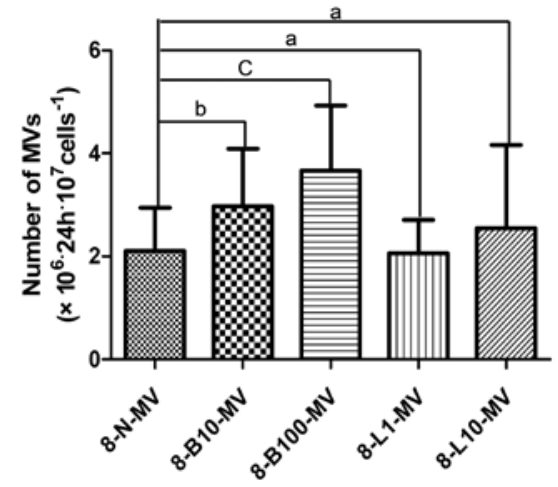

Figure 1. Quantification of MM MVs from the RPMI-8226 cell line. MV events were counted by flow cytometry and compared among the different samples. ${ }^{\mathrm{a}} \mathrm{P}>0.05,{ }^{\mathrm{b}} \mathrm{P}<0.05,{ }^{\mathrm{c}} \mathrm{P}<0.01$. MM, multiple myeloma; MM MVs, microvesicles derived from MM cells. Treatment groups: N-MV (PBS), B10-MV (10 nM bortezomib), B100-MV (100 nM bortezomib), L1-MV (1 $\mu \mathrm{M}$ lenalidomide), and L10-MV (10 $\mu \mathrm{M}$ lenalidomide).

Statistical analysis. All data are expressed as the means \pm SEM of at least three experiments. Differences among groups were analyzed with one-way ANOVA, and differences were considered statistically significant at $\mathrm{P}<0.05$.

\section{Results}

The number of MM MVS was altered following bortezomib treatment. MM cells had a high rate of MV shedding; significantly more MVs were observed in the bortezomib groups (B10-MV and B100-MV) than in the control group, but the number of MVs was not significantly altered by lenalidomide treatment (Fig. 1).

Drug treatment significantly decreases levels of VEGF, IL-6 and $b F G F$ in MM MVs. MM cells in the log phase of growth were seeded at $5 \times 10^{5}$ cells $/ \mathrm{ml}$ and were randomly divided into 5 groups according to treatment: PBS (control), $10 \mathrm{nM}$ bortezomib, $100 \mathrm{nM}$ bortezomib, $1 \mu \mathrm{M}$ lenalidomide, or $10 \mu \mathrm{M}$ lenalidomide. After $24 \mathrm{~h}, \mathrm{MVs}$ were isolated from the various conditioned media, and RNA was extracted from MVs. The PCR results showed that both bortezomib and lenalidomide treatment significantly decreased the gene expression of VEGF, IL-6 and bFGF in MVs compared with those treated with PBS, but no difference was observed between the bortezomib and lenalidomide groups (Fig. 2A).

Internalization of MM MVs alters the level of pro-angiogenic factors in HUVECs. HUVECs were randomly divided into 6 groups and co-cultured with the different MM MVs (as above) or with PBS (control group) for $24 \mathrm{~h}$. qPCR and ELISA assays were used to examine the expression levels of VEGF, IL-6 and bFGF in HUVECs and in the conditioned medium, respectively. Both qPCR (Fig. 2B) and ELISA (Fig. 2C) results showed that these cytokines were significantly higher in the normal MV-treated HUVECs than in the PBS group. Moreover, VEGF, IL-6 and bFGF expression levels in all drug-treated-MV groups were significantly decreased compared with those in the normal-MV group (Fig. 2B and C). 


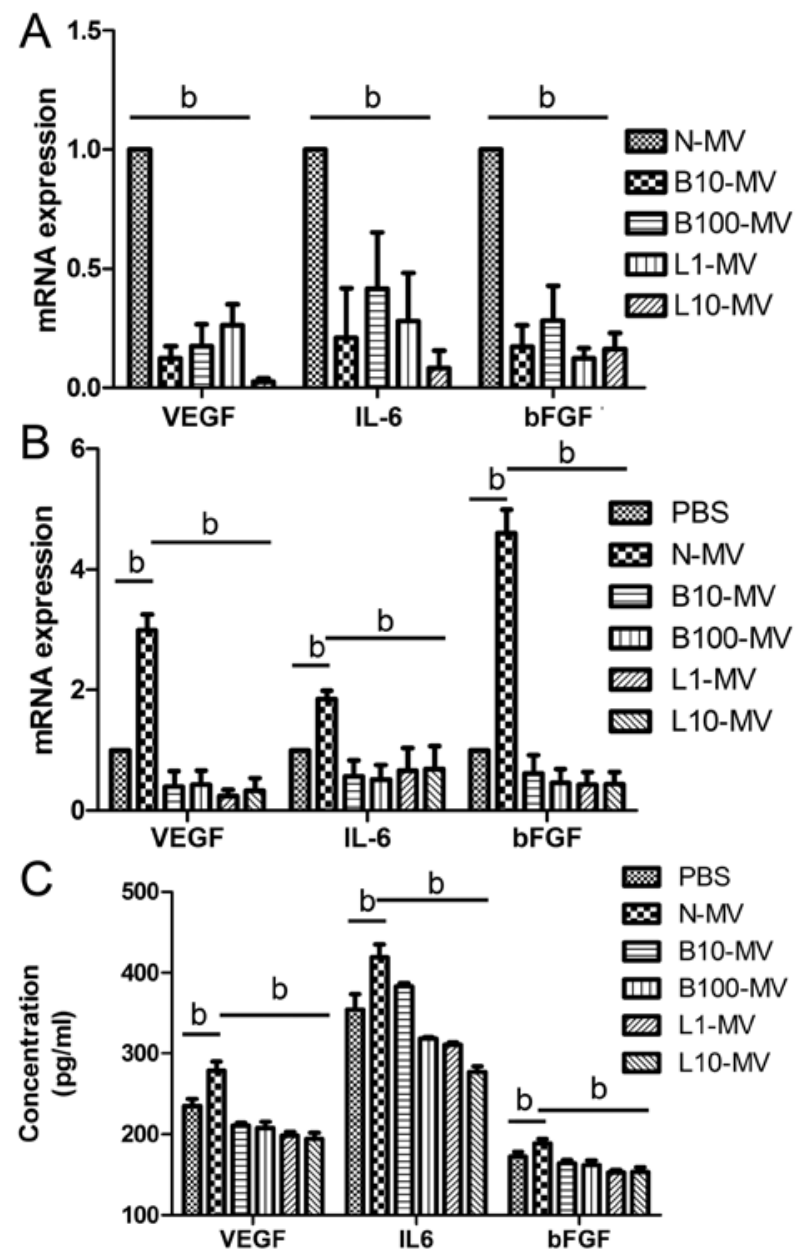

Figure 2. Drug treatment leads to decreased pro-angiogenic factor expression. (A) The mRNA expression levels of VEGF, IL-6 and bFGF were significantly decreased in the drug-treated microvesicles (MVs) compared with those in the normal MVs. After incubation with drug-treated MM MVs for $24 \mathrm{~h}$, reduced expression levels of VEGF, IL-6 and bFGF were found in the cells (B) and supernatant $(C)$ of HUVECs. ${ }^{b} \mathrm{P}<0.05$. VEGF, vascular endothelial growth factor; IL-6, interleukin 6; bFGF, basic fibroblast growth factor; HUVECs, human umbilical vein endothelial cells; MVs, microvesicles; MM, multiple myeloma; MM MVs, MVs derived from MM cells. Treatment groups: PBS (cells treated only with PBS), N-MV (PBS), B10-MV (10 nM bortezomib), B100-MV (100 nM bortezomib), L1-MV (1 $\mu \mathrm{M}$ lenalidomide), and L10-MV (10 $\mu \mathrm{M}$ lenalidomide).

Normal MVs (N-MVs) promote the migration, invasion and tube formation of HUVECs, while drug-treated MVs (DMVs) inhibit angiogenesis. The angiogenic properties of MM MVs were evaluated as their ability to induce HUVEC migration, invasion and tubulogenesis. Cell migration was tested using a wound healing assay; after treatment with N-MVs for $24 \mathrm{~h}$, HUVEC migration towards the scratched area was significantly improved. As shown in Fig. 3A, N-MVs $(10 \mu \mathrm{g} / \mathrm{ml})$ induced $55.71 \%(\mathrm{P}<0.05)$ of wound recovery compared with $44.95 \%$ in the PBS group. Compared with N-MVs, the DMVs decreased cell motility, although the B100-MV and L1-MV groups were not statistically significantly different (Fig. 3A). Next, the invasive ability of HUVECs was demonstrated by the Transwell invasion system. In this experiment, different MM MVs were used as chemoattractants. N-MVs promoted cell invasion through Matrigel, but cell invasion was significantly inhibited by the 4 groups of DMVs compared with that of the N-MV group (Fig. 3B). Finally, the angiogenic activity of MVs was investigated by in vitro tube formation assay. Tubulogenesis was qualified by counting the number of closed tubes observed at 6 and $24 \mathrm{~h}$. At $6 \mathrm{~h}$, no significant effects were observed in the N-MVs group compared with the medium control, but all DMV groups significantly suppressed the tube formation activity of HUVECs compared with that of the N-MV group (Fig. 3C). At $24 \mathrm{~h}$, the results showed that N-MVs significantly promoted the tube formation of HUVECs, but tube formation activity was significantly suppressed by the presence of DMVs (Fig. 3C).

Normal MVs (N-MVs) increase NF- $\kappa B$ activation in HUVECs, while drug-treated MVs (DMVs) reduce $N F-\kappa B$ activation. The expression of the NF- $\mathrm{B}$ p 65 subunit was determined using qPCR. The expression of NF- $\kappa \mathrm{B}$ p65 was increased in the N-MV group compared with the control group and was significantly decreased in all DMVs groups compared with the N-MV group (Fig. 4A). Then, the expression levels of p65 in the total protein and p-p65 in the nuclear protein of HUVECs were analyzed by western blot analysis, and the ratio of p-p65 to p65 was calculated. As shown in Fig. 4B, the protein expression level and the mean optical density of p-p65 were higher in the N-MV group than in the control group. However, all DMV treatments caused a reduction in the protein expression levels and the mean densities of p-p65 in nuclear proteins compared with those in the N-MV-treated group. However, p65 exhibited no significant differences among the MV groups. As shown in Fig. 4C, the same tendency was found in the immunofluorescence microscopy assay; p65 translocation was greatly increased in the N-MV group and reduced in the DMV groups. Our data suggested that N-MVs significantly increased NF- $\kappa \mathrm{B}$ activation in HUVECs, whereas it was decreased by DMVs. These data supported the regulation of $N F-\kappa B$ activity in target HUVECs by different MVs.

\section{Discussion}

Multiple myeloma (MM) is a B-cell neoplasm. Selective supportive conditions of the bone marrow (BM) microenvironment, especially BM angiogenesis (26), are involved in the development of MM. Microvesicles (MVs), also called microparticles, range in size from 100 to $1000 \mathrm{~nm}$ in diameter, are formed by the outward blebbing of the plasma membrane and are known as important players in the cell-to-cell communication that occurs in normal physiology and in pathological conditions such as the angiogenic process (10-14). A recent study has shown that MM-derived MVs (MM MVs) are internalized by endothelial cells and promote angiogenesis (14) in vitro and in vivo. Our results in the present study also indicated that MVs from normal cultured myeloma cells can induce angiogenesis in vitro.

The NF- $\kappa \mathrm{B}$ signaling pathway is activated in myeloma under environmental stress, which plays a critical role in disease oncogenesis and progression (27). Angiogenesis is critical for the progression of multiple myeloma. NF- $\kappa \mathrm{B}$ regulates the expression of pro-angiogenic genes related to myeloma pathogenesis including VEGF and IL-6 (28). In addition, interactions between myeloma cells and the bone marrow environment are critical to $\mathrm{NF}-\kappa \mathrm{B}$ activation and myeloma 

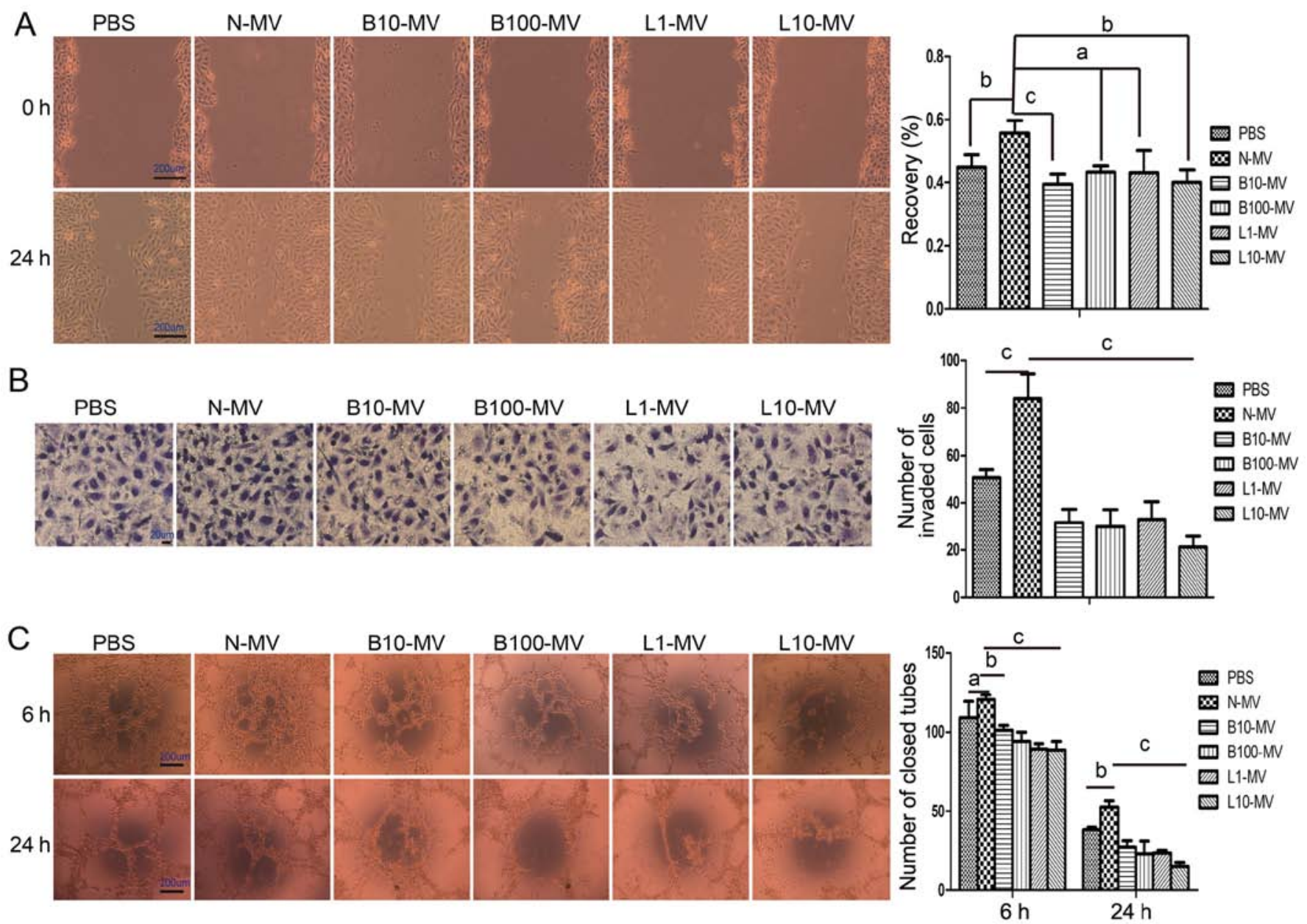

Figure 3. MM MVs (N-MVs) have angiogenic effects on HUVECs, while drug-treated MVs (DMVs) inhibit angiogenesis. (A) N-MVs increased HUVEC migration, while DMVs reduced it. The denuded area was captured (x40) at 0 and $24 \mathrm{~h}$ (left panel), and the percentage of wound recovery was calculated (right panel). (B) N-MVs promoted HUVEC invasion, while DMVs reduced it. Photomicrographs (x400) depict the invading HUVECs at the end of the experiment (left panel). The number of invading cells was counted and compared (right panel) among groups. (C) N-MVs enhanced HUVEC tubulogenesis, while DMVs inhibited it. Typical morphology of the tube-like structure formed by HUVECs (x40) is shown (left panel). A quantitative measurement of the closed tubes was performed, and the results are shown (right panel). (A-C) Data are presented as the means \pm SEM from triplicate experiments. ${ }^{a} \mathrm{P}>0.05,{ }^{b} \mathrm{P}<0.05$, ${ }^{\mathrm{C}} \mathrm{P}<0.01$. MVs, microvesicles; MM, multiple myeloma; MM MVs, MVs derived from MM cells; HUVECs, human umbilical vein endothelial cells.Treatment groups: PBS (HUVECs treated only with PBS), N-MV (PBS), B10-MV (10 nM bortezomib), B100-MV (100 nM bortezomib), L1-MV (1 $\mu$ M lenalidomide), and L10-MV (10 $\mu \mathrm{M}$ lenalidomide).

pathogenesis. MM-derived MVs are acknowledged for their role in the cell-cell communication between MM cells and the bone marrow microenvironment, and several drugs that are effective in the treatment of multiple myeloma, including bortezomib, thalidomide, and lenalidomide, have been found to block NF- $\mathrm{BB}$ activation (18-20).

MVs are released from different cell types under both normal and stressed conditions. Their biological activity depends on the origin of the cells, the content they carry and the environmental conditions of their release, which may have beneficial or harmful effects. The microenvironment can regulate their release and the amount and quality of their cargo. Herein, we studied various differences in MV populations, content and angiogenic impact under different conditions of normal culture and drug treatment (bortezomib or lenalidomide). In this study, the number of MM MVs was substantially increased in a dose-dependent manner when MM cells were treated with bortezomib compared to the number of MVs in untreated control cells. In contrast, exposing cells to lenalidomide did not significantly change the number of MVs. We speculate that these phenomena may be due to differences between cytotoxic drugs, which induce tumor cell apoptosis and anti-angiogenic agents, which inhibit endothelial cells instead of tumor cells and do not cause apoptosis. This is consistent with previous reports $(21,22)$. The number of tumorderived microparticles (TMPs) was significantly increased in breast carcinoma cells exposed to paclitaxel chemotherapy compared to the number in untreated tumor cells (21), but treatment with anti-VEGF-A neutralizing antibodies, which is a cytostatic drug, did not lead to a significant change in the number of TMPs (22).

We were surprised to find that MVs from cells exposed to bortezomib and lenalidomide both exhibited reduced angiogenic potential compared to MVs from control cells, as evidenced by the reduced migration, invasion and tubulogenesis of HUVECs exposed to DMVs. EVs have increased levels of angiogenic cytokines, such as $\operatorname{VEGF}(29,30)$ and IL-6 (31), which play a crucial role in MM pathogenesis and 

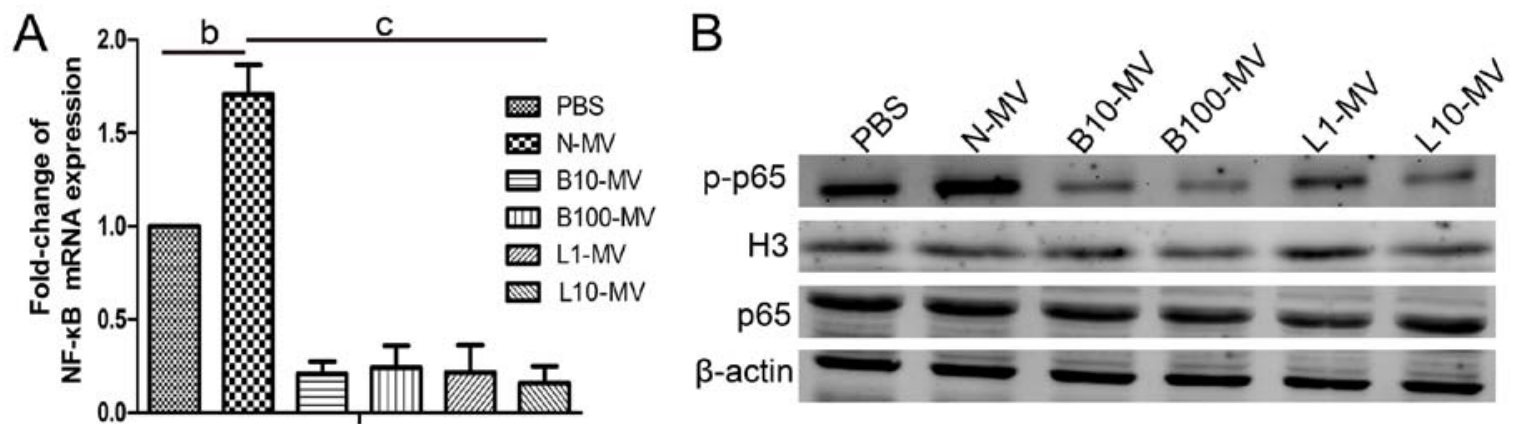

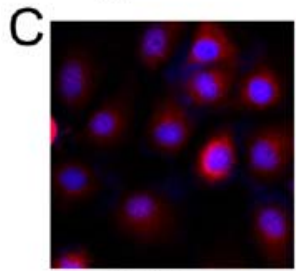

PBS

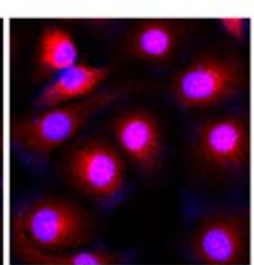

N-MV

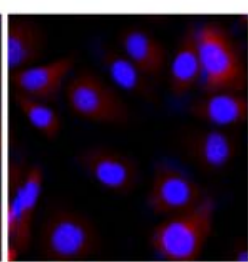

B10-MV

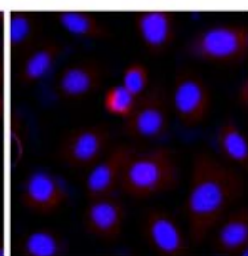

B100-MV

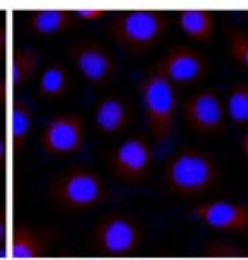

L1-MV

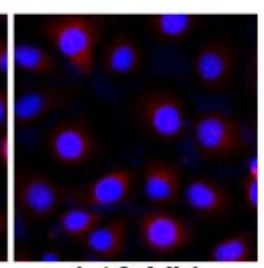

L10-MV

Figure 4. Effect of MM MVs on the activity of the NF- $\kappa$ B signaling pathway in HUVECs. N-MV significantly increased NF- $\kappa \mathrm{B}$ activation, whereas DMVs inhibited it. (A) HUVECs $\left(5 \times 10^{4}\right.$ cells/ml) were co-cultured with different MM MVs (10 $\left.\mu \mathrm{g} / \mathrm{ml}\right)$ for $24 \mathrm{~h}$ and then analyzed by qPCR. (B) Representative western blot membranes showing the expression levels of NF- $\kappa \mathrm{B}$ p-p65 and $\mathrm{H} 3$ in nuclear protein and of NF- $\mathrm{B}$ p65 and $\beta$-actin in total protein. $\beta$-actin and H3 were used as internal controls. (C) Representative immunofluorescence microphotographs showing the translocation of NF- $\mathrm{B}$ p65 in HUVECs (x100). Red (representative of the area that contains p65) and blue (representative of the nucleus part that is DAPI conjugated) area images were overlaid, creating a purple fluorescence in areas of colocalization. In the control group, the NF- $\mathrm{B}$ p 65 subunit was predominantly localized in the cytoplasm. Cells stimulated with N-MVs showed a significant translocation of p65 to the cell nucleus. In cells co-cultured with DMVs, NF- $\kappa \mathrm{B}$ p65 was significantly retained in the cytoplasm. MVs, microvesicles; MM, multiple myeloma; MM MVs, MVs derived from MM cells; HUVECs, human umbilical vein endothelial cells. Treatment groups: PBS (HUVECs treated only with PBS), N-MV (PBS), B10-MV (10 nM bortezomib), B100-MV (100 nM bortezomib), L1-MV (1 $\mu$ M lenalidomide), and L10-MV (10 $\mu \mathrm{M}$ lenalidomide)

tumor progression. In our study, the MVs derived from normal cultured MM cells contained high levels of VEGF, IL-6 and bFGF. However, we demonstrated that the expression levels of these pro-angiogenic factors were decreased in MVs from bortezomib- and lenalidomide-treated cells, thus we speculate that these drugs alter the angiogenic activity of MVs by reducing their content of angiogenic factors.

We also demonstrated that after co-culture with MVs from bortezomib- or lenalidomide-treated MM cells, endothelial cells had reduced expression levels of VEGF, IL-6 and VEGF. A previous study demonstrated that MM MVs exposed to bortezomib had lower expression levels of angiogenic factors, which limited the proliferation and migration of these endothelial cells (23). Similarly, another study showed that the expression level of VEGF-A was decreased in TMPs from breast carcinoma cells exposed to anti-VEGF-A antibody and thereby reduced the angiogenic potential (22). However, the impact of anti-angiogenic therapy with lenalidomide has not been elucidated; moreover, the effects of bortezomib- or lenalidomide-exposed MVs on the NF- $\kappa \mathrm{B}$ activity of endothelial cells have not been reported. Here, we showed that the expression levels of angiogenic factors were reduced in MVs from myeloma cells exposed to either bortezomib or lenalidomide. Furthermore, both bortezomib$\mathrm{MV}$ - and lenalidomide-MV-treated endothelial cells had reduced expression levels of VEGF, IL-6 and bFGF compared with those of the N-MV-treated group. Consequently, these MVs exhibited a reduced angiogenic ability, as evaluated by migration, invasion and tubulogenesis assays. Furthermore, NF- $\kappa \mathrm{B}$ was activated in endothelial cells co-cultured with MVs from untreated cells, while NF- $\kappa$ B activity was reduced in endothelial cells incubated with MVs from bortezomib- and lenalidomide-exposed cells.
EVs can modulate angiogenesis by stimulating or inhibiting it, highly depending on the content of EV and the expression of surface molecules, which are highly regulated by the stimulation of EV production (7). Many mechanisms are involved in the modulation of angiogenesis by EVs. For example, transfer of proteins such as $\operatorname{VEGF}(11,32), \mathrm{bFGF}(32)$ and regulation of signaling pathways, such as PI3K (10),

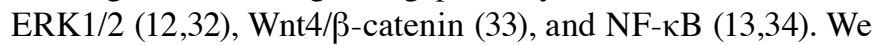
speculate that drug-treated MVs can inhibit angiogenesis by the following mechanisms. i) Microvesicles contain VEGF, IL-6 and bFGF mRNA, and they can release these contents after internalization by endothelial cells. Drug-exposed MVs had decreased mRNA expression levels of VEGF, IL-6 and bFGF, leading to a reduced expression of VEGF, IL-6 and bFGF mRNA in endothelial cells and a decreased secretion of these pro-angiogenic factors. ii) Microvesicles act as exogenous substances that can be internalized by endothelial cells and activate $\mathrm{NF}-\kappa \mathrm{B}$, resulting in increased VEGF, IL- 6 and bFGF mRNA expression and protein secretion in endothelial cells. Microvesicles exposed to drugs can inhibit NF- $\kappa \mathrm{B}$ after fusion with endothelial cells and thereby lead to decreased expression levels of angiogenic factors. However, It remains unclear how the drug-treated MVs inhibit NF- $\mathrm{B}$ activation and to what extent the inhibition of angiogenesis by drugtreated MVs can be attributed to NF- $\kappa \mathrm{B}$ inhibition. Overall, our data suggest that there are additional mechanisms worthy of further investigation.

\section{Acknowledgements}

Not applicable. 


\section{Funding}

This study was funded by the National Natural Science Foundation of China (no. 81500163).

\section{Availability of data and materials}

The datasets used during the present study are available from the corresponding author upon reasonable request.

\section{Authors' contributions}

LS, JML and HMG conceived and designed the study. HMG, LY and ZYN performed the experiments. HMG wrote the paper. LS, XJL and JML reviewed and edited the manuscript. All authors read and approved the manuscript and agree to be accountable for all aspects of the research in ensuring that the accuracy or integrity of any part of the work are appropriately investigated and resolved.

\section{Ethics approval and consent to participate}

Not applicable.

\section{Consent for publication}

Not applicable.

\section{Competing interests}

The authors declare that they have no competing interests.

\section{References}

1. Kumar SK, Rajkumar SV, Dispenzieri A, Lacy MQ, Hayman SR Buadi FK, Zeldenrust SR, Dingli D, Russell SJ, Lust JA, et al: Improved survival in multiple myeloma and the impact of novel therapies. Blood 111: 2516-2520, 2008.

2. Gkotzamanidou M, Christoulas D, Souliotis VL, Papatheodorou A, Dimopoulos MA and Terpos E: Angiogenic cytokines profile in smoldering multiple myeloma: No difference compared to MGUS but altered compared to symptomatic myeloma. Med Sci Monit 19: 1188-1194, 2013.

3. Accardi F, Toscani D, Bolzoni M, Dalla Palma B, Aversa F and Giuliani N: Mechanism of action of bortezomib and the new proteasome inhibitors on myeloma cells and the bone microenvironment: Impact on myeloma-induced alterations of bone remodeling. BioMed Res Int 2015: 172458, 2015.

4. Matthes T, Manfroi B, Zeller A, Dunand-Sauthier I, Bogen B and Huard B: Autocrine amplification of immature myeloid cells by IL-6 in multiple myeloma-infiltrated bone marrow. Leukemia 29: 1882-1890, 2015.

5. Dankbar B, Padró T, Leo R, Feldmann B, Kropff M, Mesters RM, Serve H, Berdel WE and Kienast J: Vascular endothelial growth factor and interleukin- 6 in paracrine tumor-stromal cell interactions in multiple myeloma. Blood 95: 2630-2636, 2000.

6. van der Pol E, Böing AN, Harrison P, Sturk A and Nieuwland R: Classification, functions, and clinical relevance of extracellular vesicles. Pharmacol Rev 64: 676-705, 2012.

7. Todorova D, Simoncini S, Lacroix R, Sabatier F and DignatGeorge F: Extracellular Vesicles in Angiogenesis. Circ Res 120: 1658-1673, 2017.

8. EL Andaloussi S, Mäger I, Breakefield XO and Wood MJ: Extracellular vesicles: Biology and emerging therapeutic opportunities. Nat Rev Drug Discov 12: 347-357, 2013.

9. Canella A, Harshman SW, Radomska HS, Freitas MA and Pichiorri F: The potential diagnostic power of extracellular vesicle analysis for multiple myeloma. Expert Rev Mol Diagn 16 277-284, 2016
10. Deregibus MC, Cantaluppi V, Calogero R, Lo Iacono M, Tetta C, Biancone L, Bruno S, Bussolati B and Camussi G: Endothelial progenitor cell derived microvesicles activate an angiogenic program in endothelial cells by a horizontal transfer of mRNA. Blood 110: 2440-2448, 2007.

11. Zou X, Gu D, Xing X, Cheng Z, Gong D, Zhang G and Zhu Y: Human mesenchymal stromal cell-derived extracellular vesicles alleviate renal ischemic reperfusion injury and enhance angiogenesis in rats. Am J Transl Res 8: 4289-4299, 2016.

12. Lombardo G, Dentelli P, Togliatto G, Rosso A, Gili M, Gallo S, Deregibus MC, Camussi G and Brizzi MF: Activated Stat5 trafficking via endothelial cell-derived extracellular vesicles controls IL-3 pro-angiogenic paracrine action. Sci Rep 6: 25689, 2016.

13. Anderson JD, Johansson HJ, Graham CS, Vesterlund M, Pham MT, Bramlett CS, Montgomery EN, Mellema MS, Bardini RL, Contreras Z, et al: Comprehensive proteomic analysis of mesenchymal stem cell exosomes reveals modulation of angiogenesis via nuclear factor-kappab signaling. Stem Cells 34: 601-613, 2016.

14. Liu Y, Zhu XJ, Zeng C, Wu PH, Wang HX, Chen ZC and Li QB: Microvesicles secreted from human multiple myeloma cells promote angiogenesis. Acta Pharmacol Sin 35: 230-238, 2014.

15. Li ZW, Chen H, Campbell RA, Bonavida B and Berenson JR: NF-kappaB in the pathogenesis and treatment of multiple myeloma. Curr Opin Hematol 15: 391-399, 2008.

16. Chilov D, Kukk E, Taira S, Jeltsch M, Kaukonen J, Palotie A, Joukov V and Alitalo K: Genomic organization of human and mouse genes for vascular endothelial growth factor C. J Biol Chem 272: 25176-25183, 1997.

17. Cramer M, Nagy I, Murphy BJ, Gassmann M, Hottiger MO, Georgiev $\mathrm{O}$ and Schaffner W: NF-kappaB contributes to transcription of placenta growth factor and interacts with metal responsive transcription factor-1 in hypoxic human cells. Biol Chem 386: 865-872, 2005.

18. Nencioni A, Grünebach F, Patrone F, Ballestrero A and Brossart P: Proteasome inhibitors: Antitumor effects and beyond. Leukemia 21: 30-36, 2007.

19. De Luisi A, Ferrucci A, Coluccia AM, Ria R, Moschetta M, de Luca E, Pieroni L, Maffia M, Urbani A, Di Pietro G, et al: Lenalidomide restrains motility and overangiogenic potential of bone marrow endothelial cells in patients with active multiple myeloma. Clin Cancer Res 17: 1935-1946, 2011.

20. Singhal S and Mehta J: Lenalidomide in myeloma. Curr Treat Options Oncol 8: 154-163, 2007.

21. Fremder E, Munster M, Aharon A, Miller V, Gingis-Velitski S, Voloshin T, Alishekevitz D, Bril R, Scherer SJ, Loven D, et al: Tumor-derived microparticles induce bone marrow-derived cell mobilization and tumor homing: A process regulated by osteopontin. Int J Cancer 135: 270-281, 2014.

22. Munster M, Fremder E, Miller V, Ben-Tsedek N, Davidi S, Scherer SJ and Shaked Y: Anti-VEGF-A affects the angiogenic properties of tumor-derived microparticles. PLoS One 9: e95983, 2014.

23. Zarfati M, Katz T, Avivi I, Brenner B and Aharon A: PO-45 - The role of microvesicles in multiple myeloma progression. Thromb Res 140 (Suppl 1): S193, 2016.

24. Sun L, Wang HX, Zhu XJ, Wu PH, Chen WQ, Zou P, Li QB and Chen ZC: Serum deprivation elevates the levels of microvesicles with different size distributions and selectively enriched proteins in human myeloma cells in vitro. Acta Pharmacol Sin 35: 381-393, 2014.

25. Ma GF, Chen S, Yin L, Gao XD and Yao WB: Exendin-4 ameliorates oxidized-LDL-induced inhibition of macrophage migration in vitro via the NF- $\kappa \mathrm{B}$ pathway. Acta Pharmacol Sin 35: 195-202, 2014.

26. Ribatti D and Vacca A: The role of microenvironment in tumor angiogenesis. Genes Nutr 3: 29-34, 2008.

27. Klein B: Positioning NK-kappaB in multiple myeloma. Blood 115: 3422-3424, 2010.

28. Podar K and Anderson KC: Inhibition of VEGF signaling pathways in multiple myeloma and other malignancies. Cell Cycle 6: 538-542, 2007.

29. Thompson CA, Purushothaman A, Ramani VC, Vlodavsky I and Sanderson RD: Heparanase regulates secretion, composition, and function of tumor cell-derived exosomes. J Biol Chem 288: 10093-10099, 2013.

30. Mineo M, Garfield SH, Taverna S, Flugy A, De Leo G, Alessandro R and Kohn EC: Exosomes released by K562 chronic myeloid leukemia cells promote angiogenesis in a Src-dependent fashion. Angiogenesis 15: 33-45, 2012. 
31. Roccaro AM, Sacco A, Maiso P, Azab AK, Tai YT, Reagan M, Azab F, Flores LM, Campigotto F, Weller E, et al: BM mesenchymal stromal cell-derived exosomes facilitate multiple myeloma progression. J Clin Invest 123: 1542-1555, 2013.

32. Brill A, Dashevsky O, Rivo J, Gozal Y and Varon D: Plateletderived microparticles induce angiogenesis and stimulate post-ischemic revascularization. Cardiovasc Res 67: 30-38, 2005.
33. Zhang B, Wu X, Zhang X, Sun Y, Yan Y, Shi H, Zhu Y, Wu L, Pan $\mathrm{Z}$, Zhu W, et al: Human umbilical cord mesenchymal stem cell exosomes enhance angiogenesis through the Wnt $4 / \beta$-catenin pathway. Stem Cells Transl Med 4: 513-522, 2015.

34. Shabbir A, Cox A, Rodriguez-Menocal L, Salgado M and Van Badiavas E: Mesenchymal stem cell exosomes induce proliferation and migration of normal and chronic wound fibroblasts, and enhance angiogenesis in vitro. Stem Cells Dev 24: 1635-1647, 2015. 